\title{
Measurement of the Proton's Neutral Weak Magnetic Form Factor
}

\author{
B. Mueller, ${ }^{1}$ D. H. Beck, ${ }^{2}$ E. J. Beise,${ }^{3}$ E. Candell,${ }^{4} *$ L. Cardman,,${ }^{2,}$ R. Carr, ${ }^{1}$ R. C. DiBari, ${ }^{4}$ G. Dodson, ${ }^{5}$ K. Dow,${ }^{5}$ \\ F. Duncan, ${ }^{3}$ M. Farkhondeh, ${ }^{5}$ B. W. Filippone,,${ }^{1}$ T. Forest,${ }^{2}$ H. Gao, ${ }^{2, \ddagger}$ W. Korsch,${ }^{1,}{ }^{\S}$ S. Kowalski, ${ }^{5}$ A. Lung, ${ }^{3}$ R. D. \\ McKeown, ${ }^{1}$ R. Mohring, ${ }^{3}$ J. Napolitano, ${ }^{4}$ D. Nilsson, ${ }^{2}$ M. Pitt, ${ }^{1, \|}$ N. Simicevic, ${ }^{2, \| l}$ B. Terburg, ${ }^{2}$ and S. P. Wells ${ }^{5, \pi}$ \\ (SAMPLE Collaboration) \\ ${ }^{1}$ Kellogg Radiation Laboratory, California Institute of Technology, Pasadena, California 91125 \\ ${ }^{2}$ University of Illinois at Urbana-Champaign, Urbana, Illinois 61801 \\ ${ }^{3}$ University of Maryland, College Park, Maryland 20742 \\ ${ }^{4}$ Rensselaer Polytechnic Institute, Troy, New York 12180 \\ ${ }^{5}$ Bates Linear Accelerator Center, Laboratory for Nuclear Science and Department of Physics, \\ Massachusetts Institute of Technology, Cambridge, Massachusetts 02139
}

(Received 24 February 1997)

\begin{abstract}
We report the first measurement of the parity-violating asymmetry in elastic electron scattering from the proton. The asymmetry depends on the neutral weak magnetic form factor of the proton which contains new information on the contribution of strange quark-antiquark pairs to the magnetic moment of the proton. We obtain the value $G_{M}^{Z}=0.34 \pm 0.09 \pm 0.04 \pm 0.05$ n.m. at $Q^{2}=0.1(\mathrm{GeV} / c)^{2}$. [S0031-9007(97)03181-5]
\end{abstract}

PACS numbers: 13.60.Fz, 11.30.Er, 13.40.Gp, 14.20.Dh

The measurement of strange quark-antiquark $(\bar{s} s)$ effects in the nucleon offers a unique window to study the effects of the $\bar{q} q$ "sea" at low momentum transfers. This information is an important clue to the dynamical effects of QCD that are responsible for form factors in the nonperturbative regime, and may lead to new insight into the origins of these effects.

It has been shown [1] that the neutral weak current can be used to determine the $\bar{s} s$ contributions to nucleon form factors. The magnetic moment is one important nucleon property that can be studied in this fashion. The neutral weak magnetic form factor of the proton can be measured in parity-violating electron scattering, [2], thus providing information on the $\bar{s} s$ content of the nucleon's magnetic moment. In this Letter, we report the first such measurement and obtain the first direct experimental data relevant to determination of the strange magnetic moment of the proton.

To lowest order (tree-level), the neutral weak magnetic form factor of the proton $G_{M}^{Z}$ can be related to nucleon electromagnetic form factors and a contribution from strange quarks:

$$
G_{M}^{Z}=\frac{1}{4}\left(G_{M}^{p}-G_{M}^{n}\right)-\sin ^{2} \theta_{W} G_{M}^{p}-\frac{1}{4} G_{M}^{s},
$$

where $G_{M}^{p}$ and $G_{M}^{n}$ are the (electromagnetic) nucleon magnetic form factors, and $\theta_{W}$ is the weak mixing angle. (Note that the weak mixing angle has recently been determined [3] with high precision: $\sin ^{2} \theta_{W}\left(M_{Z}\right)=0.2315 \pm$ 0.0004.) Electroweak radiative corrections must be applied to the coefficients in Eq. (1), which have been computed in Ref. [4]. Then measurement of the neutral weak form factor $G_{M}^{Z}$ will allow (after combination with the well known electromagnetic form factors) determination of the strange magnetic form factor $G_{M}^{s}$. There has been a variety of theoretical predictions for $G_{M}^{s}$ in the limit of zero momentum transfer $\left(\equiv \mu_{s}\right)$ over the last few years, and summaries of them are presented in Refs. [5] and [6]. The typical magnitude is $-0.3 \mathrm{n} . \mathrm{m}$. (nuclear magnetons), although the predictions range from -0.73 to +0.42 n.m.

As mentioned above, the quantity $G_{M}^{Z}$ for the proton can be measured via elastic parity-violating electron scattering at backward angles [2]. The difference in cross sections for right and left handed incident electrons arises from interference of the electromagnetic and neutral weak amplitudes, and so contains products of electromagnetic and neutral weak form factors. The expression for elastic scattering from the proton is given by

$$
\begin{aligned}
A= & \frac{\sigma_{R}-\sigma_{L}}{\sigma_{R}+\sigma_{L}} \\
= & {\left[\frac{-G_{F} Q^{2}}{\pi \alpha \sqrt{2}}\right] } \\
& \times \frac{\varepsilon G_{E}^{\gamma} G_{E}^{Z}+\tau G_{M}^{\gamma} G_{M}^{Z}-\frac{1}{2}\left(1-4 \sin ^{2} \theta_{W}\right) \varepsilon^{\prime} G_{M}^{\gamma} G_{A}^{Z}}{\varepsilon\left(G_{E}^{\gamma}\right)^{2}+\tau\left(G_{M}^{\gamma}\right)^{2}},
\end{aligned}
$$

where $\varepsilon, \tau$, and $\varepsilon^{\prime}=\sqrt{\tau(1+\tau)\left(1-\varepsilon^{2}\right)}$ are kinematic quantities, and $Q^{2}>0$ is the four-momentum transfer [5]. In addition to the electric and magnetic neutral weak form factors, $G_{E}^{Z}$ and $G_{M}^{Z}$, the numerator of this expression also contains the neutral weak axial form factor:

$$
G_{A}^{Z}=-\frac{1}{2}\left(1+R_{A}\right) G_{A}+\frac{1}{2} G_{A}^{s},
$$

where we take $G_{A}=1.2601$ from neutron beta decay [3], $G_{A}^{s}=\Delta s \sim-0.1$ is from polarized deep inelastic scattering [7], and $R_{A} \sim-0.34$ is the axial radiative 
correction [4,5]. This axial radiative correction is quite uncertain and we assign a $100 \%$ uncertainty to this correction. The uncertainty due to the $G_{A}^{S}$ term is about an order of magnitude smaller than the radiative correction uncertainty.

The SAMPLE experiment measures the parity-violating asymmetry in the elastic scattering of $200 \mathrm{MeV}$ incident electrons at backward angles with an average $Q^{2} \simeq$ $0.1(\mathrm{GeV} / c)^{2}$. For $G_{M}^{s}=0$, the value of $G_{M}^{Z}$ (at $Q^{2}=$ $\left.0.1 \mathrm{GeV}^{2}\right)$ is 0.40 and the expected asymmetry in the SAMPLE experiment is $-7.2 \times 10^{-6}$ or $-7.2 \mathrm{ppm}$; for $G_{M}^{s}=-0.3$ n.m., $G_{M}^{Z}=0.48$ and we would then expect $A=-8.3 \mathrm{ppm}$. At these kinematics the axial term contributes about $20 \%$ of the asymmetry, so the uncertainty due to the axial term is $\pm 0.7 \mathrm{ppm}$.

The experiment is performed at the MIT/Bates Linear Accelerator Center using a $200 \mathrm{MeV}$ polarized electron beam incident on a liquid hydrogen target. The scattered electrons are detected in a large solid angle $(\sim 2 \mathrm{sr})$ air Cerenkov detector similar in design to that used in Ref. [8]. The detector covers the angular region $130^{\circ}<$ $\theta<170^{\circ}$ corresponding to the range of momentum transfers $0.10<Q^{2}<0.11(\mathrm{GeV} / c)^{2}$.

The liquid hydrogen target is $40 \mathrm{~cm}$ long, and is part of a high flow-rate recirculating liquid hydrogen system with a heat exchanger to remove the $\sim 500$ watts of heat deposited by the electron beam. Studies of the performance [9] of the target indicate that with $40 \mu \mathrm{A}$ of beam the target can be maintained as a subcooled liquid and that density fluctuations are less than $0.1 \%$. The fluctuations in the detected signal are then dominated by counting statistics of the scattered particles.

The detector consists of ten large mirrors, each with ellipsoidal curvature to focus the Cerenkov light onto one of ten photomultiplier tubes. Each photomultiplier is 8 in. in diameter and is shielded from the target and room background by a cast lead shield. In addition, $2 \mathrm{~mm}$ of $\mathrm{Pb}$ on the scattering chamber wall is essential to reduce the x-ray background in the detector to a reasonable level. (This backround is due to Compton scattering of forward bremsstrahlung in the liquid hydrogen target.)

A remotely controlled light shutter can cover each photomultiplier tube for background measurements. We typically take one third of our data with shutters closed to monitor this background. We have determined that this background is soft electromagnetic radiation (a thin $\mathrm{Pb}$ shield eliminates it) and arises from showering in the target plus additional scattering from downstream of the apparatus. Electromagnetic background generated by soft bremsstrahlung will have negligible helicity dependence. (The parity-violating asymmetry is proportional to the squared momentum transfer to the proton which is extremely small.) Our measurements do verify an absence of helicity dependence in this signal (see Table I).

In addition, we study the detector signals with covers on the mirrors to analyze them for non-Cerenkov sources
TABLE I. Components of detector signal.

\begin{tabular}{ccc}
\hline \hline Source & Fraction & Asymmetry $(\mathrm{ppm})$ \\
\hline Elastic electrons & $54.8 \pm 4.0 \%$ & $-6.34 \pm 1.45 \pm 0.53$ \\
Shutter closed & $22.6 \pm 0.5 \%$ & $1.95 \pm 3.13$ (Measured) \\
$\pi$ decays & $2.3 \pm 1.4 \%$ & $<1 \mathrm{ppm}$ (Ref. [11]) \\
x rays (EM) & $20.3 \pm 3.7 \%$ & 0 \\
\hline \hline
\end{tabular}

of light. To further study these backgrounds, we developed a technique of accelerating "tracer bullets" of $\sim 0.1 \mathrm{~mA}$ peak current interspersed with very weak beam pulses with typical peak currents in the nA range. One can then use more conventional pulse counting techniques to study the Cerenkov detector response. This method clearly shows the relative fraction of scintillation light (events with only one photoelectron) relative to Cerenkov light (events with several photoelectrons). The scintillation light is due to the interaction of the residual flux of $\mathrm{x}$ rays and low energy gamma rays from the target with the air in the detector and the rate agrees with a computer simulation of this process. As indicated in Table I, this background amounts to $20.3 \pm 3.7 \%$ of our total detector signal and should have negligible helicity dependence (as discussed in the previous paragraph).

The Cerenkov light is almost totally due to elastic scattering of electrons from protons, with a small contribution due to pion decays. The pion background is rather small because the low incident beam energy $(200 \mathrm{MeV})$ is close to the production threshold. The pion yield has been calculated using known photoproduction cross sections, and the low $\pi^{+}$yield is verified by measurement of the rate of delayed signals from muon decays just after the electron beam pulse ends. In addition, we used a NaI detector behind a mirror in coincidence to check the spectrum of scattered electrons and compare with computer simulations. The observed elastic scattering signal is in good agreement with expectations.

The incident electron beam is pulsed at $600 \mathrm{~Hz}$; each detector signal is integrated over the $\sim 15 \mu \mathrm{sec}$ of every beam pulse and digitized. The beam intensity is similarly integrated and digitized. The ratio of integrated detector signal to the integrated beam signal is the normalized yield which is proportional to the cross section (plus background). We then measure the helicity dependent asymmetry in the normalized yield which (after correction for background, radiative effects, and beam polarization) yields the parity-violating asymmetry $A$. All ten detectors are combined in software during the data analysis.

The polarized electron source is a GaAs photoemission source. The laser beam that is incident on the GaAs crystal is circularly polarized by a $\lambda / 4$ Pockels cell. The electron beam helicity is rapidly reversed by changing the voltage on the Pockels cell to reverse the circular polarization of the light. The helicity is randomly chosen for each of ten consecutive beam pulses and then the complement helicities are used for the next ten pulses. 
The asymmetry in the normalized yields is computed for "pulse pairs" separated by $1 / 60$ of a second to minimize systematic errors. Each pulse pair asymmetry corresponds to a measurement of the parity-violating cross section asymmetry. These are then combined in 30 minute runs corresponding to typically $0.5 \times 10^{6}$ measurements. An active feedback system [10] keeps the helicity correlations in the beam intensity averaged to zero to better than $1 \mathrm{ppm}$.

The beam helicity can be manually reversed by rotating a $\lambda / 2$ plate which reverses the helicity of the light (and the beam) relative to all electronic signals. We denote this setting as "reverse" as opposed to the "normal" setting; a real parity violation signal will appear to change sign under this "slow reversal." Electronic crosstalk and other effects will not change under slow reversal, so this is an important test that our signal is not some spurious systematic effect.

The electron polarization is measured using a Moller apparatus on the beam line and is typically $35 \%$. A Wien spin rotator located at the exit of the polarized source is used to rotate the spin for investigation of transverse spin asymmetries and minimize their effects during parity violation measurements. Based on these studies, the effect of transverse components of electron polarization to the observed parity violation signal is determined to be negligible.

The beam position and angle at the target in both transverse dimensions ( $x$ and $y$ ), the beam energy, and the "halo" of the beam are continuously monitored for every beam pulse. If a helicity correlation is present, then we can correct the detector normalized yield asymmetries to remove its effect. In practice, these corrections are generally less than $1 \mathrm{ppm}$ per run and approximately average to zero over many runs. Other properties, such as the width of the beam, are studied during special runs to verify the absence of helicity correlations.

We have acquired a significant data sample from two runs: one in fall 1995 and one in spring 1996. Combining the data in each of the two data sets and computing the physics asymmetry, we obtain the asymmetries shown in Fig. 1. The corrections to the raw measured asymmetry are the background effects given in Table I $(1.82 \pm 0.13)$, the correction for (internal and external) bremsstrahlung radiation $(1.16 \pm 0.01)$, and the beam polarization $[1 /(0.348 \pm 0.015)]$. These are combined to give an overall multiplicative correction of $6.07 \pm 0.51$. We have assumed that the shutter closed background and the $\mathrm{x}$-ray background light have zero asymmetry, as expected for soft electromagnetic processes. Combining the asymmetries from the two data sets yields the value for the parity-violating asymmetry

$$
A=-6.34 \pm 1.45 \pm 0.53 \mathrm{ppm},
$$

where the first uncertainty is statistical and the second is the estimated systematic error. The neutral weak

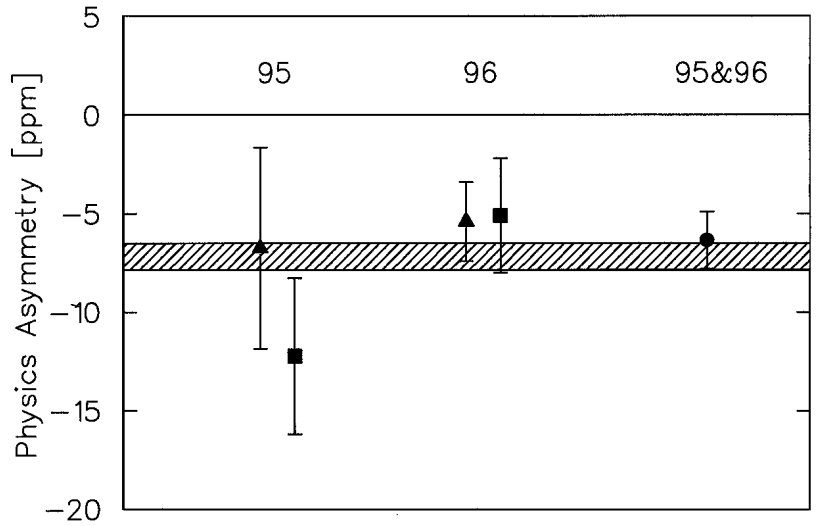

FIG. 1. Results for the parity-violating asymmetry measured in the 1995 and 1996 running periods. For each running period we display the result for the normal (squares) and reverse (triangles) settings of slow helicity reversal, which are in good agreement. The combined result for both helicity states and both running periods is also shown (circle). The error bars include statistical errors only. The hatched region indicates the asymmetry band (due to the uncertain axial radiative correction) for $\mu_{s}=G_{M}^{s}=0$.

magnetic form factor derived from this asymmetry is

$$
G_{M}^{Z}\left(Q^{2}=0.1 \mathrm{GeV}^{2}\right)=0.34 \pm 0.09 \pm 0.04 \pm 0.05 \text { n.m. },
$$

where the last uncertainty is due to the axial radiative correction and other minor form factor uncertainties associated with extracting $G_{M}^{Z}$ from the measured asymmetry. In the absence of a strange quark contribution we expect $G_{M}^{Z}=0.40$ n.m.; our measurement of $G_{M}^{Z}$ thus corresponds to a value of $G_{M}^{s}\left(Q^{2}=0.1 \mathrm{GeV}^{2}\right)=$ $+0.23 \pm 0.37 \pm 0.15 \pm 0.19$ n.m.

If we do not assume the shutter closed background to be identically zero but rather use the measured shutter closed asymmetry (see Table I), then the result for $A$ becomes $-7.38 \pm 2.12 \pm 0.66 \mathrm{ppm}$ where the statistical error is increased by the shutter closed measurement. This value is in good agreement with that obtained above.

In the future, for the full hydrogen data set we project that the statistical error on $G_{M}^{Z}$ will be reduced to about $0.05 \mathrm{~nm}$. (We have collected only about $20 \%$ of the total data sample in these two runs.) Additional running with deuterium will further reduce the uncertainty in $G_{M}^{Z}$ by greatly reducing the error associated with the axial radiative correction [12]. The deuterium asymmetry contains the same fraction of axial correction but is almost completely insensitive to $G_{M}^{s}$. As a result the ratio $A_{p} / A_{D}$ has the same sensitivity to $G_{M}^{S}$ but almost no sensitivity to $R_{A}$.

Additional experiments to explore other features of neutral weak currents and strange form factors of the nucleon are planned at Mainz (MAMI-B) [13] and TJNAF (formerly known as CEBAF) [14]. These experiments will provide a new and unique window on the quark structure of the nucleon, and hopefully will provide important 
information towards a more complete understanding of nucleon structure in the context of QCD.

The persistent efforts of the staff of the MIT/Bates facility to provide high quality beam and improve the experiment are gratefully acknowledged. Financial support for the construction of the Moller apparatus from CEBAF is acknowledged. This work was supported by NSF Grants No. PHY-9420470 (Caltech), No. PHY-9420787 (Illinois), No. PHY-9457906/PHY-9229690 (Maryland), No. PHY-9507412 (RPI), and DOE Cooperative Agreement No. DE-FC02-94ER40818 (MIT/Bates).

\footnotetext{
*Deceased.

†Present address: Thomas Jefferson National Accelerator Facility, Newport News, VA 23606.

*Present address: Physics Division, Argonne National Laboratory, Argonne, IL 60439.

${ }^{\S}$ Present address: Department of Physics and Astronomy, University of Kentucky, Lexington, KY 40506.

"Present address: Department of Physics, Virginia Polytechnic Institute and State University, Blacksburg, VA 24061-0435.

IPresent address: Department of Physics, Louisiana Tech
}

University, Ruston, LA 71270.

[1] D. Kaplan and A. Manohar, Nucl. Phys. B310, 527 (1988).

[2] R. D. McKeown, Phys. Lett. B 219, 140 (1989); D. H. Beck, Phys. Rev. D 39, 3248 (1989).

[3] Particle Data Group, R. M. Barnett et al., Phys. Rev. D 54, 1 (1996).

[4] M. J. Musolf and B. R. Holstein, Phys. Lett. B 242, 461 (1990).

[5] M. J. Musolf et al., Phys. Rep. 239, 1 (1994).

[6] E. J. Beise et al., in Proceedings of SPIN96 Symposium (Report No. nucl-ex/9610011).

[7] K. Abe et al., Phys. Rev. Lett. 74, 346 (1995).

[8] W. Heil et al., Nucl. Phys. B327, 1 (1989).

[9] E. J. Beise et al., Nucl. Instrum. Methods Phys. Res., Sect. A 378, 383 (1996).

[10] P. A. Souder et al., Phys. Rev. Lett. 65, 694 (1990).

[11] S.-P. Li, E. M. Henley, and W.-Y.P. Hwang, Ann. Phys. (N.Y.) 143, 372 (1982).

[12] Bates experiment 94-11 (M. Pitt and E. J. Beise, spokespersons).

[13] Mainz proposal A4/1-93 94-11 (D. von Harrach, spokesperson).

[14] TJNAF experiments E91-017 (D. Beck, spokesperson); E91-010 (P. Souder and J. Finn, spokespersons); E91-004 (E. J. Beise, spokesperson). 